\title{
DIGITAL ASSESSMENT OF DEPTH OF INVASION IN MELANOMA USING DIFFERENT IMMUNOHISTOCHEMICAL STAINS
}

\author{
Leila ALI ${ }^{1,2 \bowtie}$, Reza ALAGHEHBANDAN ${ }^{3}$, Valentin T. MOLDOVAN ${ }^{1,2}$, Diana DEREWICZ ${ }^{2,4}$, \\ Anca M. CORICOVAC ${ }^{2}$, Octav GINGHINA ${ }^{2}$, Maria SAJIN ${ }^{2,5}$, Mariana COSTACHE ${ }^{2,5}$ \\ ${ }^{1}$ Department of Pathology, National Institute of Pathology „Victor Babes“, Bucharest, Romania \\ ${ }^{2}$ University of Medicine and Pharmacy „Carol Davila“, Bucharest, Romania \\ ${ }^{3}$ Department of Pathology, Faculty of Medicine, University of British Columbia, Royal Columbian Hospital, \\ Vancouver, BC, Canada \\ 4"M. S. Curie" Clinical Emergency Hospital for Children, Bucharest, Romania \\ ${ }^{5}$ Department of Pathology, University Emergency Hospital, Bucharest, Romania
}

Received 13 Febr 2020, Corrections received 01 Apr 2020, Accepted 02 May 2020

https://doi.org/10.31688/ABMU.2020.55.2.12

\section{Abstract}

Introduction. The most important histologic prognostic factors for melanoma remain depth of invasion and mitotic rate. In a digital era, precision (in terms of reproducibility and inter laboratories repeatability), as well as image evidence, are new qualitative parameters. The objective of the study was to evaluate in a retrospective analysis any significant difference among the Breslow digital assessments performed using two different immunohistochemical stains and the gold standard, hematoxylin eosin stain.

Material and methods. We evaluated 130 primary cutaneous melanomas diagnosed in a two-year period (2016-2017) with known Breslow scale. Immunohistochemistry staining (SOX10 and Melan A) was perform followed by slide scanning. Aperio Imagescope and StataCorp software were used for data acquisition and statics.

\section{Résumé}

Evaluation digitale de l'invasion en profondeur des mélanomes, à l'aide de différents marqueurs immunohistochimiques

Introduction. Les facteurs histologiques pronostiques les plus importants du mélanome restent la profondeur d'invasion et le taux mitotique. Dans l'ère digitale, la précision (en termes de reproductibilité et de répétabilité inter laboratoires), ainsi que les preuves des images, sont de nouveaux paramètres qualitatifs.

L'objectif de l'étude a été d'évaluer, dans une analyse rétrospective, toute différence significative entre les évaluations numériques de Breslow effectuées en utilisant deux colorations immunohistochimiques différentes et l'étalon d'or, la coloration à l'éosine d'hématoxyline.

Matériel et méthodes. Nous avons évalué 130 mélanomes cutanés primaires diagnostiqués pour 
Results. No significantly statistic differences were recorded between groups in terms of Breslow values ( $\mathrm{p}=0.98$, Kruskal-Wallis test).

Conclusions. All three stains perform similar in evaluating depth of invasion regardless of the method used to quantify it. The pathologist should consider using an accurate and precise method like a digital measurement technique for daily reports.

Keywords: melanoma, image analysis, Breslow depth, immunohistochemistry.

\author{
Abbreviations list \\ $\mathrm{HE}$ - hematoxylin-eosin \\ IHC - immunohistochemistry \\ $\mathrm{ABC}$ - avidin-biotin complex \\ WSI - whole slide image \\ SPSS - Statistical Package for the Social Sciences \\ LDH - lactate dehydrogenase
}

\section{INTRODUCTION}

Melanoma is the most fatal type of skin cancer, causing about 50,000 deaths per year'. The gold standard in diagnosing and staging melanoma consists of histopathological tissue analysis, along with Breslow thickness and lymph nodes assessment. The increasing efforts in public education have led to a shift in stage on the moment of diagnosis, nowadays $70 \%$ of the invasive lesions presenting as T1 stage tumors ${ }^{2}$. This fact, together with the 2018 American Joint Committee on Cancer (AJCC) revision in the definitions of $\mathrm{T} 1 \mathrm{a}$ and $\mathrm{T} 1 \mathrm{~b}$ categories ${ }^{3}$, increase the need for accuracy when measuring thickness. The augmentation in computational power and improvement in image analysis $s^{4-7}$ have made possible the emergence of new analytical perspectives, including the possibility of implementing digital measurements. une période de deux ans (2016-2017) avec l'échelle de Breslow connue. Une coloration immunohistochimique (SOX10 et MelanA) a été effectuée suivie de la scanographie des lamelles. Les logiciels Aperio Imagescope et StataCorp ont été utilisés pour l'acquisition de données et la statique.

Résultats. Aucune différence statistiquement significative n'a été enregistrée entre les groupes en termes de valeurs de Breslow ( $p=0,98$, test de Kruskal-Wallis).

Conclusions. Les trois taches ont des performances similaires dans l'évaluation de la profondeur d'invasion, quelle que soit la méthode utilisée pour la quantifier. Le pathologiste devrait envisager d'utiliser une méthode exacte et précise comme une technique de mesurage numérique pour les rapports quotidiens.

Mots-clés: mélanome, analyse d'image, indice de Breslow, immunohistochimie.

\section{THE OBJECTIVE OF THE STUDY}

The aim of this paper is to document a cross-comparison among digital measurements on whole scanned histological images of hematoxylin-eo$\sin (\mathrm{HE})$-, MelanA- and Sox10-stained slides in a sample of 105 primary cutaneous malignant melanomas and register any statistical significant difference.

\section{Material AND MEthods}

\section{Specimen selection}

We retrospectively selected 130 patients with primary cutaneous melanomas, diagnosed between 2016-2017 in the „Victor Babes“ National Institute of Pathology, Bucharest, Romania. All HE-stained slides of the 130 cases were subsequently reviewed by 2 experienced pathologists, to confirm the diagnosis

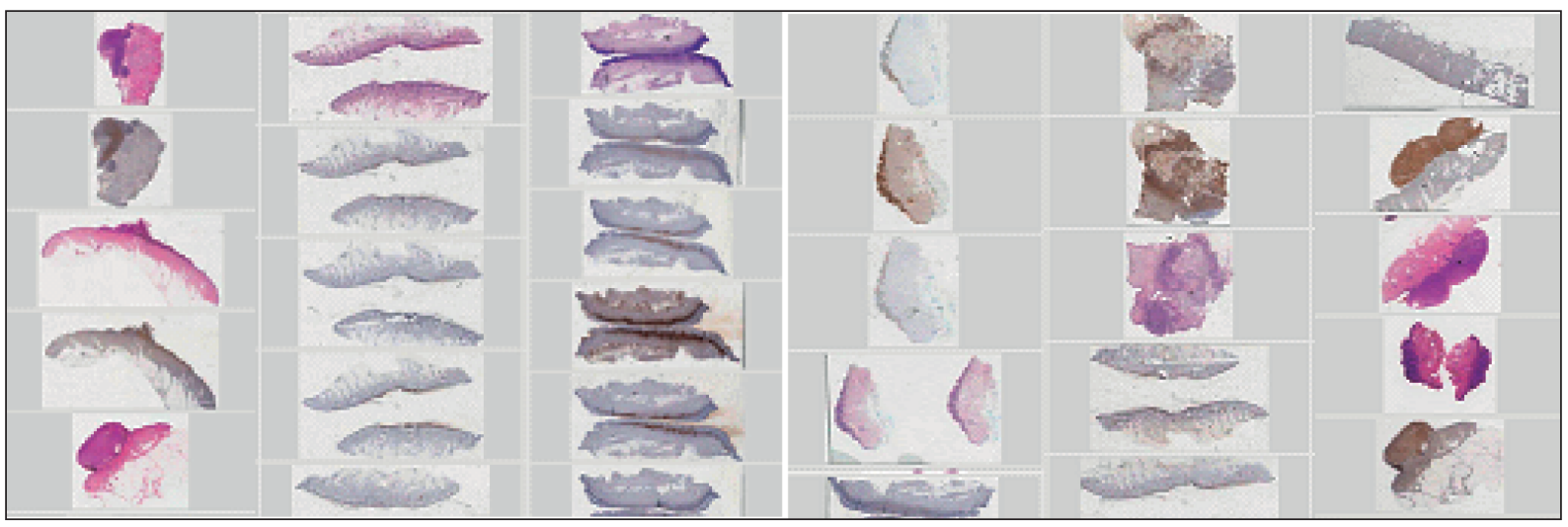

Fig. 1. Digital HE and IHC WSI archive thumbnails. 


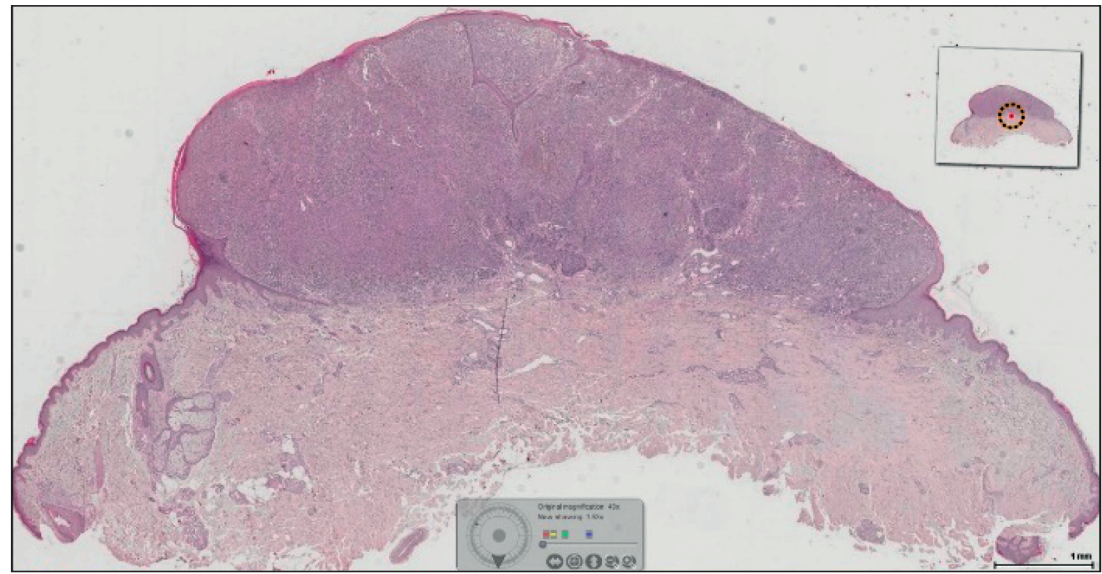

Fig. 2. The WSI of a HE-stained melanoma slide at 10x magnification allows viewing the entire tissue sample on the computer screen

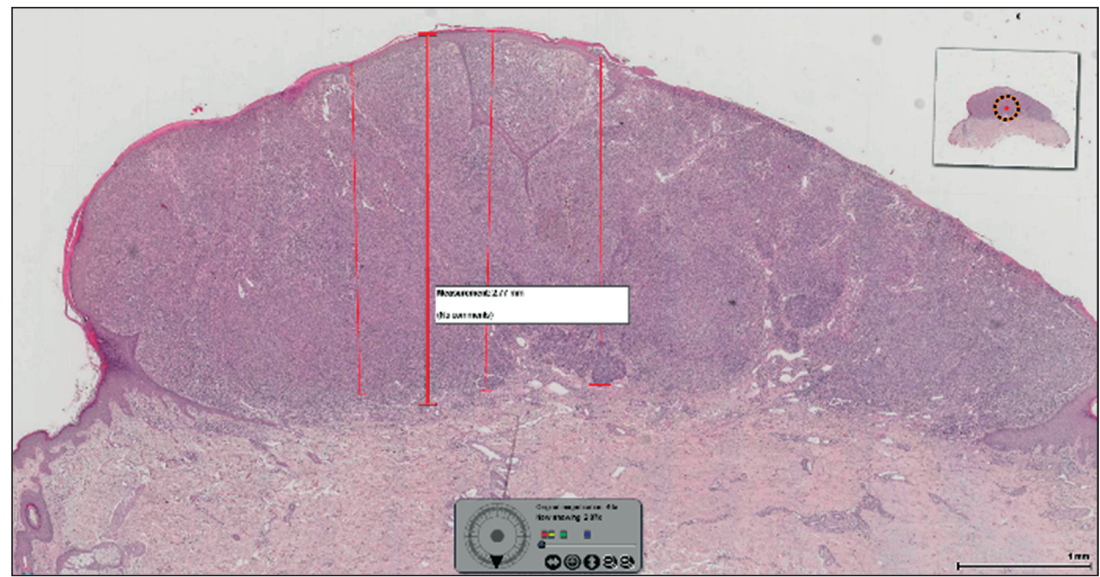

Fig. 3. Digital measurements on the WSI using AperioImageScope 12.2 software

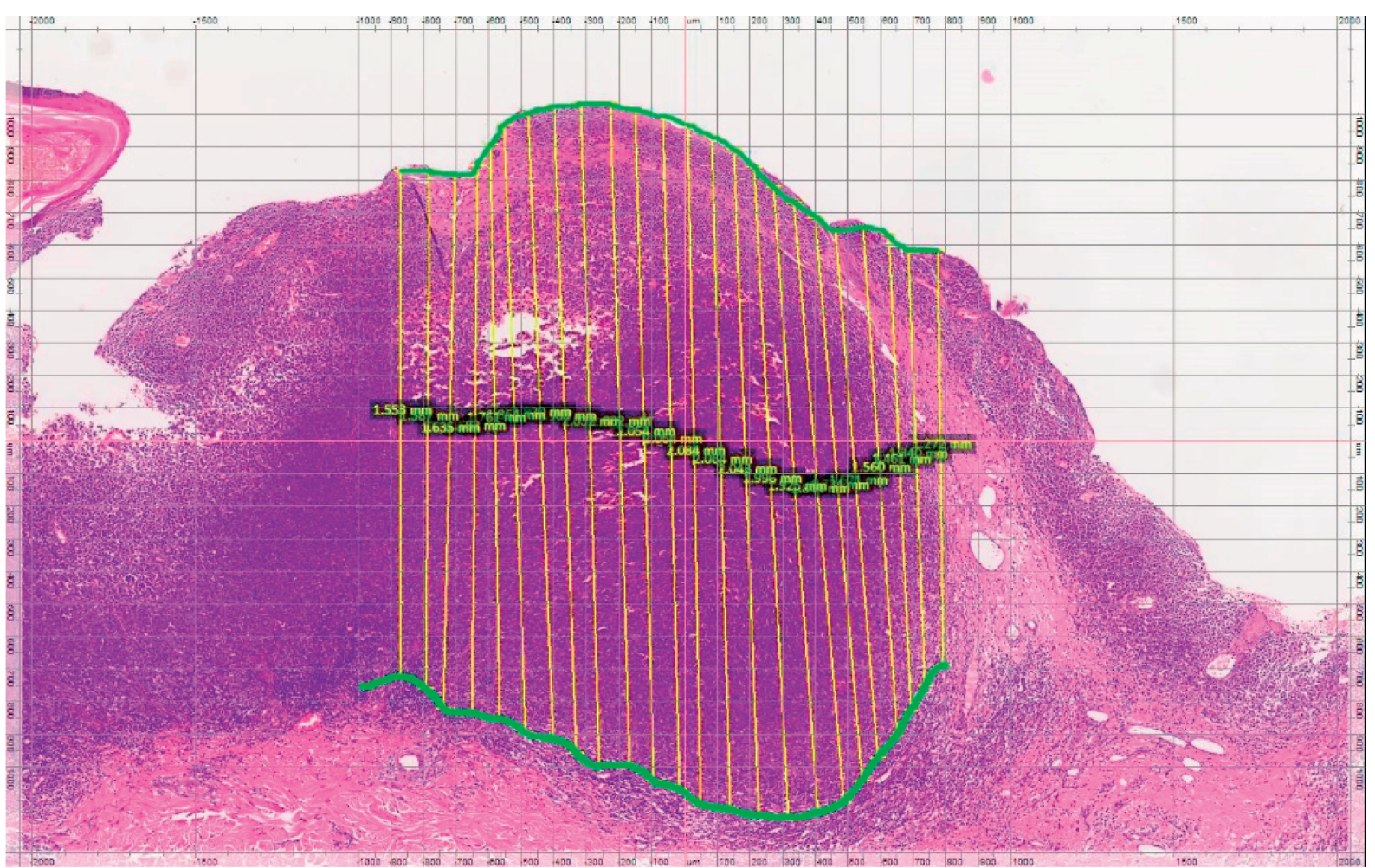

Fig.4. Using the Distance Measurement tool on a melanoma slide.

Measurement lines are placed equidistantly within the relevant area. 

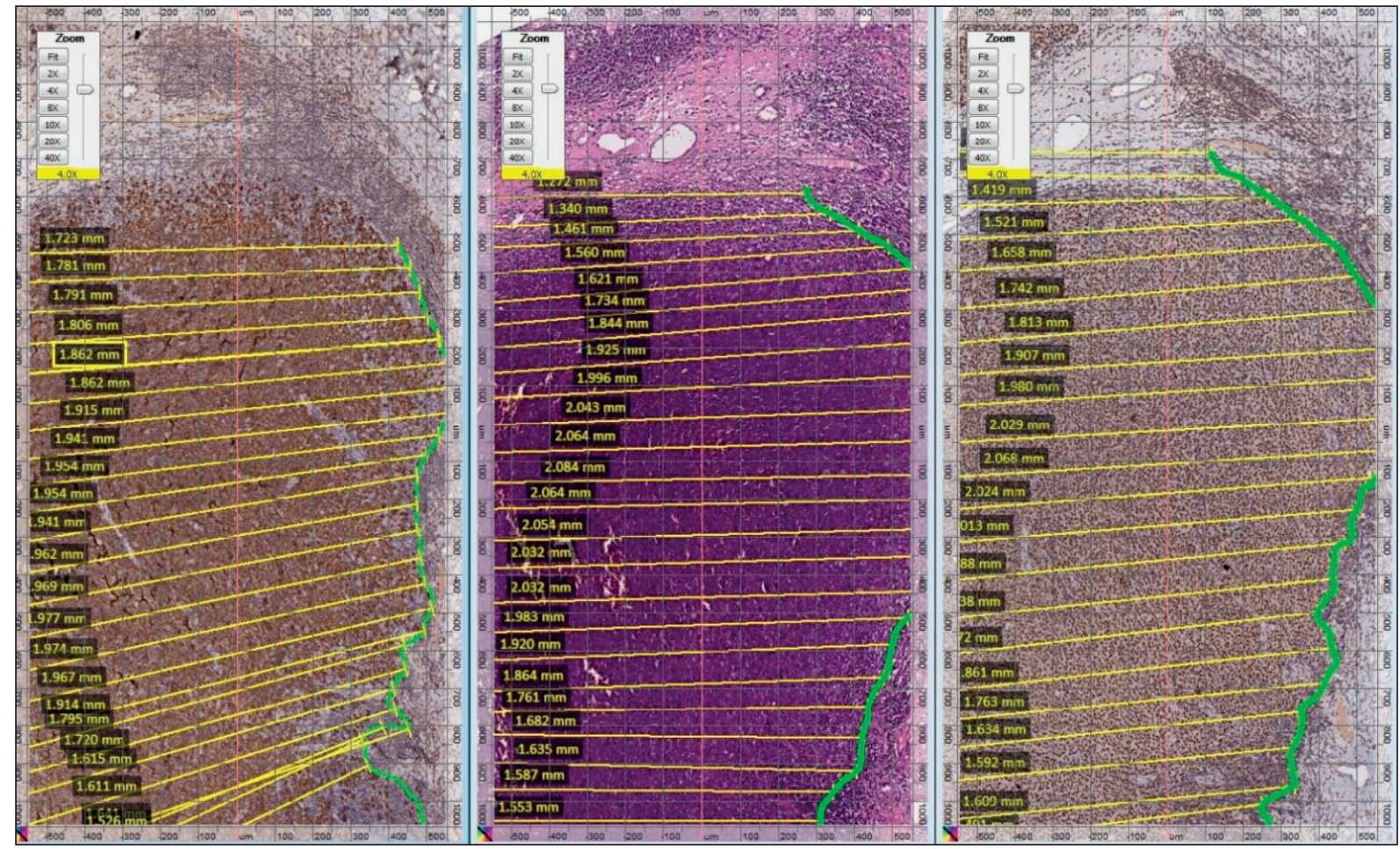

Fig 5. Performing measurements on the HE and IHC slides (40x) aligned side-by-side on the same screen.

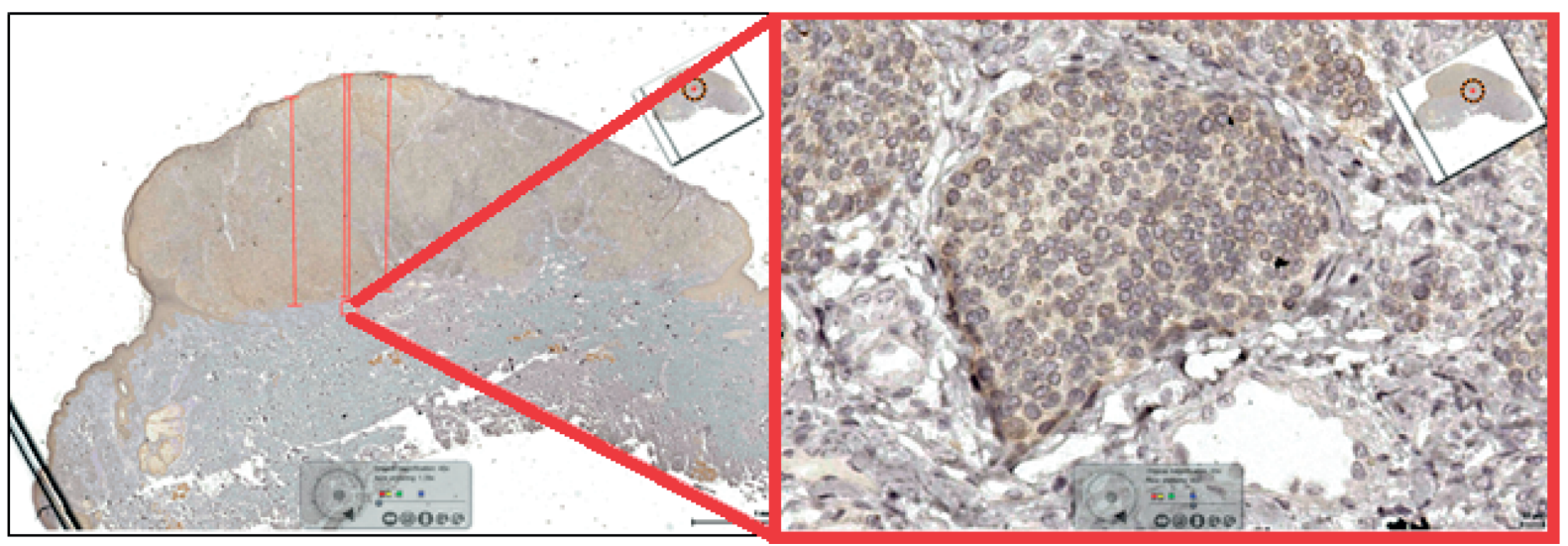

Fig 6. Aperio imaging system. a Showing an overview of a slide (10x magnification); b showing the zoomed-in image, focusing on the deepest invading cell group (400x magnification).

according to the 2018 World Health Organization diagnosis criteria ${ }^{8}$. The most representative tissue block was selected for each case, considering adequate sampling and orientation, entire tumor sections comprising maximum tumor thickness and presence of internal control structures ${ }^{9}$ for specific immunohistochemical (IHC) staining on the selected blocks. Of the 130 cases, only 105 met the selection criteria.

\section{Immunohistochemistry staining}

For each case, we performed MelanA (Roche-Ventana, A103 clone), Sox10 (Abcam, SOX10/991) and ki67 (monoclonal, Dako, Glostrup, MIB1 clone) immunostaining, using the indirect protocol for paraffin-embedded tissue sections. After pre-treatment, incubation in 3\% hydrogen peroxide and HIER antigen retrieval, we used the avidin-biotin complex (ABC) staining method. All slides expressed positive internal control (presence of MelanA/ Sox10-positive cells in the epidermal basal layer).

\section{Digitization of histological slides}

After reexamination and selection, the HE and IHC slides were scanned with an AperioAT2 Whole Slide Imaging Scanner, at a 40x magnification ${ }^{10}$. The first-time scan success rate for the slides was $96 \%$. As a result, a 2D ultra-high-resolution digital image was created for each scanned slide - the whole slide image 
Table 1. T stage distribution for each measurement, established by using corresponding Breslow value

\begin{tabular}{cccc}
\hline Stage & Digital method HE & Digital method MelanA & Digital method Sox10 \\
\hline $\mathrm{T} 1$ & 33 & 33 & 35 \\
\hline $\mathrm{T} 2$ & 18 & 18 & 16 \\
\hline $\mathrm{T} 3$ & 22 & 22 & 22 \\
\hline $\mathrm{T} 4$ & 32 & 32 & 32 \\
\hline
\end{tabular}

Table 2. Definition of Primary Tumor (T) according to AJCC 8th Edition ${ }^{3}$

\begin{tabular}{ccc}
\hline T category & Thickness & Ulceration \\
\hline Tis (melanoma in situ) & Not applicable & Not applicable \\
\hline $\mathrm{T} 1$ & $\leq 1.0 \mathrm{~mm}$ & Unknown/unspecified \\
\hline $\mathrm{T} 1 \mathrm{a}$ & $<0.8 \mathrm{~mm}$ & Without ulceration \\
\hline $\mathrm{T} 1 \mathrm{~b}$ & $<0.8 \mathrm{~mm}$ & With ulceration \\
\cline { 2 - 3 } & $0.8-1.0 \mathrm{~mm}$ & With or without ulceration \\
\hline $\mathrm{T} 2$ & $>1.0-2.0 \mathrm{~mm}$ & Unknown/unspecified \\
\hline $\mathrm{T} 2 \mathrm{a}$ & $>1.0-2.0 \mathrm{~mm}$ & Without ulceration \\
\hline $\mathrm{T} 2 \mathrm{~b}$ & $>1.0-2.0 \mathrm{~mm}$ & With ulceration \\
\hline $\mathrm{T} 3$ & $>2.0-4.0 \mathrm{~mm}$ & Unknown/unspecified \\
\hline $\mathrm{T} 3 \mathrm{a}$ & $>2.0-4.0 \mathrm{~mm}$ & Without ulceration \\
\hline $\mathrm{T} 3 \mathrm{~b}$ & $>2.0-4.0 \mathrm{~mm}$ & With ulceration \\
\hline $\mathrm{T} 4$ & $>4.0 \mathrm{~mm}$ & Unknown/unspecified \\
\hline $\mathrm{T} 4 \mathrm{a}$ & $>4.0 \mathrm{~mm}$ & Without ulceration \\
\hline $\mathrm{T} 4 \mathrm{~b}$ & $>4.0 \mathrm{~mm}$ & With ulceration \\
\hline
\end{tabular}

(WSI), which was stored on the assigned servers in a digital archive (Fig 1). The WSI may be analyzed on a computer screen, making it possible to view the image at different magnifications (Fig. 2,6), using Aperio ImageScope - Pathology Slide Viewing Software ${ }^{11}$.

\section{Data acquisition}

Digital measurements were performed, quantifying the maximum distance between the granular layer of the epidermis and the deepest point of tumor invasion on HE, MelanA and Sox10 digital slide images. The AperioImageScope 12.2 software allows the measurement of objects either by tracing perpendiculars with the Ruler tool (Fig. 3), or by using the Distance Measurement tool to measure the distance between two free-hand line annotations (Fig 4) $)^{10,11}$. We chose to operate with the former. The program also enables viewing the images on the same screen, aligning the HE and IHC slides side-by-side (Fig. 5), for improved comparison - features that are not available in optical microscopy ${ }^{12}$. For each slide, the highest value-bearing measure was elected.

\section{Data analysis}

The digitally obtained values on HE, MelanA and Sox10 slides were compared, pursuing the dissimilarity among the assessments and the impact on TNM staging, by using a paired sample t-test ${ }^{13}$ in SPSS (Statistical Package for the Social Sciences) to compare the means among the three methods. Kruskal-Wallis ${ }^{14}$ test was used for Breslow values intergroup comparisons. A p-value of 0.05 was considered statistically significant. Statistical analyses were performed using Stata/IC 16 (StataCorp).

\section{Results}

Demographic and tumor characteristics of the cohort $(n=105): 50(47.6 \%)$ patients were women, and $55(52.4 \%)$ men, resulting in an equal sex distribution; the age range was between 8 and 83 years. The most common histological subtype was nodular melanoma (45\%). Ulceration was present in $48 \%$ of the tumors.

After examining the scanned slides and performing digital measurements, we had the following results:

i) The mean tumor thickness was: 3.3037 for HE, 3.3198 for MelanA and 3.2775 for Sox10.

ii) The distribution across $T$ stage was identical for HE and MelanA, but for Sox10 two pT2 cases migrated to pT1b (Table 1). The T1 category is the most sensitive one, given that the threshold is below $1 \mathrm{~mm}$, and the measurements identified two pT1a cases in HE slides upgraded to pT1b on both MelanA and 


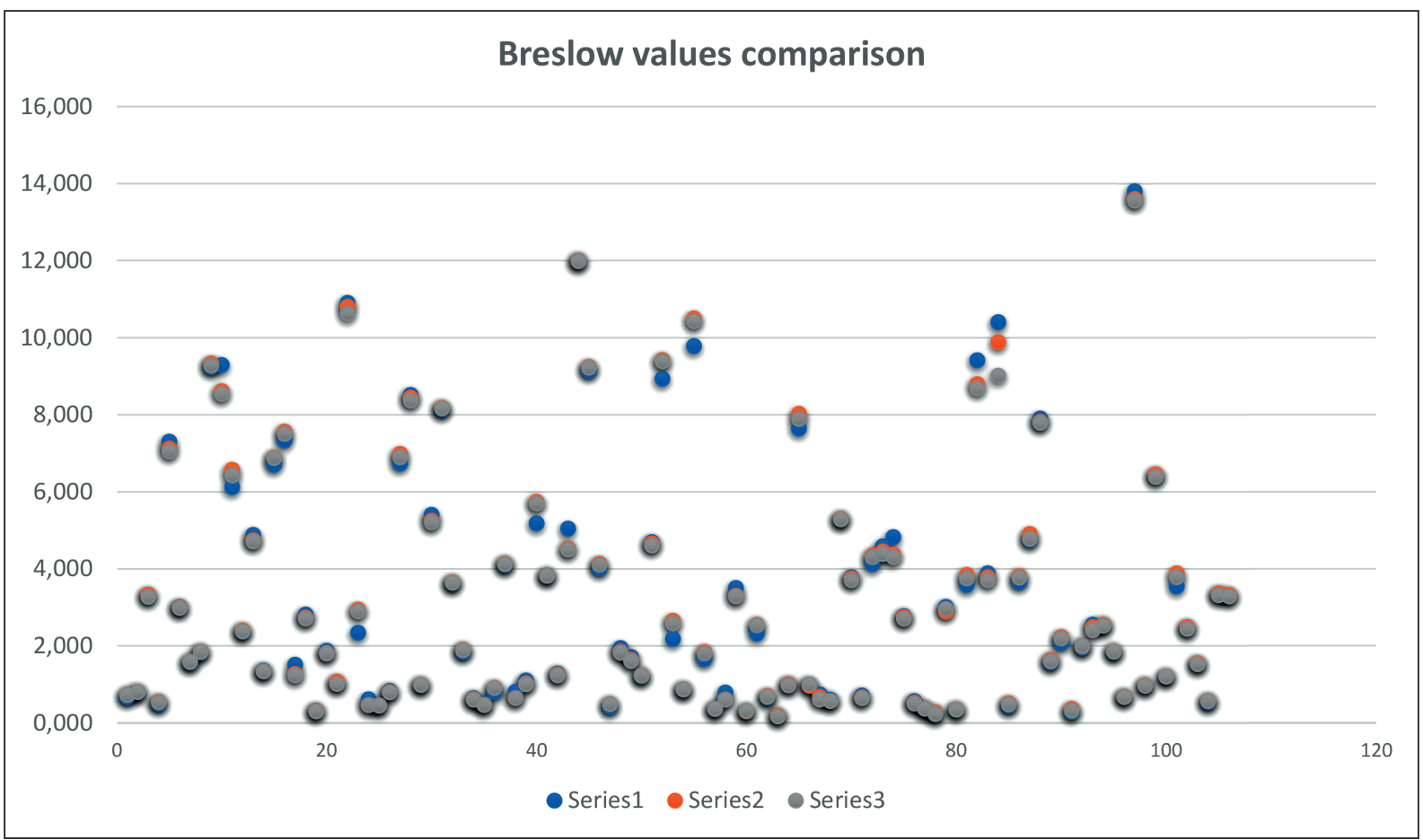

Fig 7. Breslow values comparison for each stain

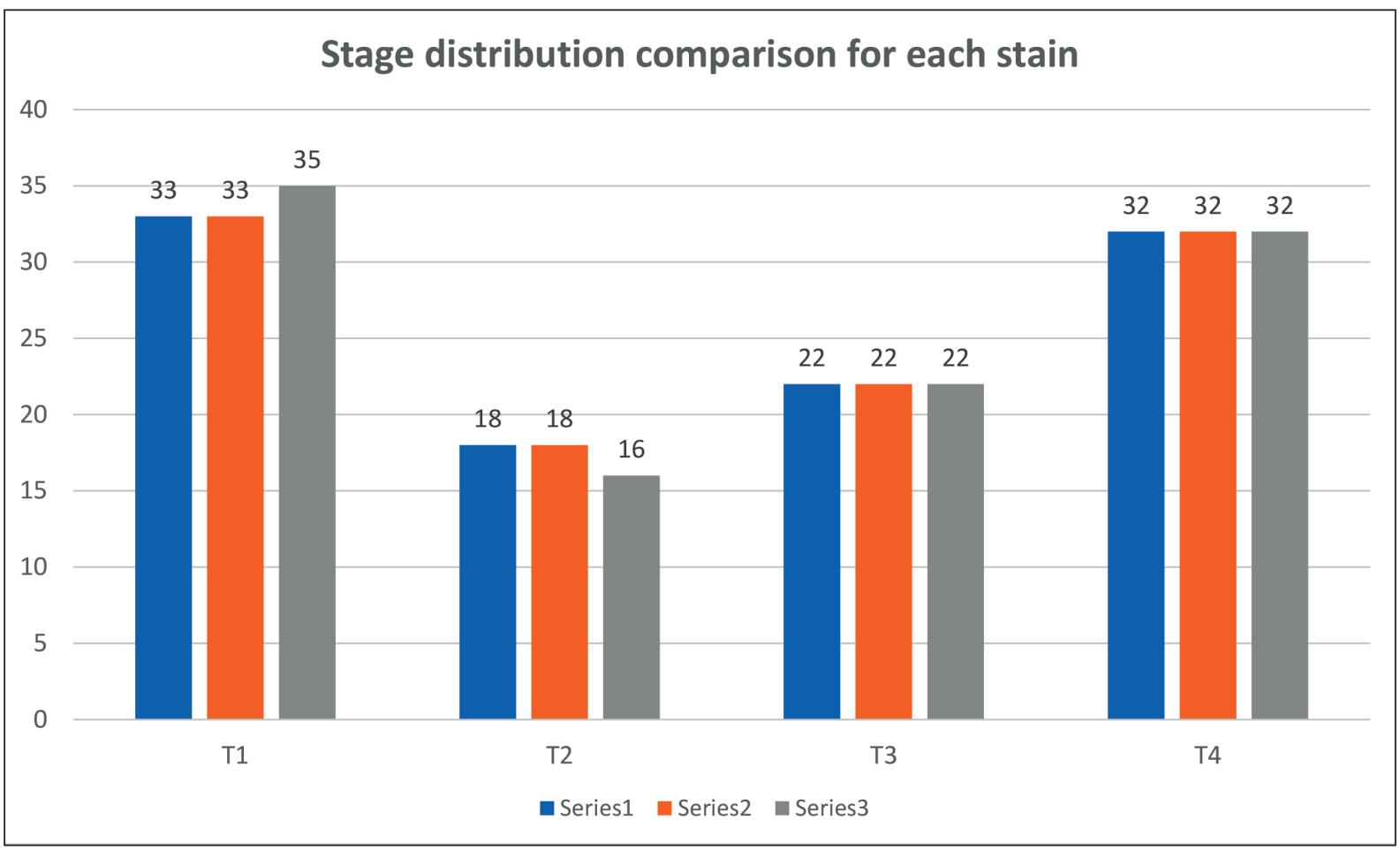

Fig 8. Stage distribution comparison for each stain

Sox10 slides, and one pT1b HE case downgraded to pTla on immuno-stains.

In $80 \%$ of the cases, the Breslow values on $\mathrm{HE}$ versus MelanA versus Sox10 were super-posable (Fig. 7). The Breslow values recorded by digital measurements on HE slides compared to MelanA slides and
Sox10 slides showed no variation in the T3-T4 categories (Fig. 8), but added a significant gradient observed in $\mathrm{T} 1$ category, with $\sim 8 \%$ of the cases being reclassified. No significantly statistic differences were recorded between groups in terms of Breslow values $(\mathrm{p}=0.98$, Kruskal-Wallis test). 


\section{Discussion}

Currently, the clinical staging (cTNM) is performed post biopsy diagnosis, and pathological staging (pTNM) follows the histological examination of the wide excision specimen. Staging must include regional lymph nodes evaluation after completing sentinel node biopsy/regional lymphadenectomy.

The staging instruments, according to the eighth edition of the AJCC melanoma staging system, are: melanoma thickness and ulceration for the $\mathrm{T}$ category; presence/absence of non-nodal regional metastases for the $\mathrm{N}$ category; site of distant metastasis for the $\mathrm{M}$ category. Also, the mitotic rate should be reported for all invasive primary melanomas, and lactate dehydrogenase (LDH) must be recorded for any M1 anatomic site of disease (Table 2).

Melanoma thickness or Breslow scale, described by Breslow in $1970^{15}$, is the most important prognostic factor ${ }^{16}$. It is performed by measuring the thickness of the tumor, from the top of the granular layer of overlying epidermis to the deepest invasive tumor cell. If the epidermis is ulcerated, instead of the granular layer, the measure concerns the thickness starting from the ulcer's base ${ }^{2}$. The consensus is to use HE staining and optical microscopy to assess the thickness. In most instances, the use of the conventional method would suffice for an accurate measurement $t^{17}$.

However, there might be cases were IHC staining would be needed in measuring the invasive component: single cell or small groups of infiltrating cells, strong host response, inflammation, fibrosis, regression, peri-adnexal extension, pre-existing nevus, verruciform architecture, neurotropic spread, etc ${ }^{3,18}$.

The purpose of the study was to compare the clinical utility in measuring the Breslow thickness on HE versus IHC (MelanA and Sox10) staining using the digital method.

The evaluation showed no significant statistical difference between Breslow thickness measurements using $\mathrm{HE}$ and Melan A and similar findings were recorded between HE and Sox10; however, mean Breslow thickness was significantly higher in Sox10 than in MelanA ( $p>0.05)$.

In the case of tumor staging on $\mathrm{HE}$ slides versus IHC, 4 lesions ended up in another T category, mainly T1 and T2 lesions.

This study suggests that HE would most likely be sufficiently reliable and accurate as IHC stains in measuring Breslow values. It should be noted that using IHC stains must be considered as an adjunct tool for final estimation/measurement, and that there are some situations when the use of a superior technique, such as digital measurement combined with IHC staining, might aid in establishing a more accurate staging, mainly in the case of pT1 lesions.

There was a significant correlation between using the 3 methods and the mitotic rate combined with the ki67 index.

A significant limitation of the study was that although the inappropriate blocks were withdrawn from the examined lot, the cutoff levels remain a strong variable, given the fact that the first slide was always stained with $\mathrm{HE}$, and the following ones were IHC slides.

\section{Conclusion}

Although HE-stained slides proved to be, in most cases, as accurate as IHC stains in measuring Breslow values, the study brings to attention the contribution of computer-assisted histopathological analysis in establishing an accurate appraisal for Breslow thickness in pT1 stage tumors. Using computer-assisted image analysis, we were able to perform exact measurements even in the case of infra-millimetric values. The data demonstrated that the lesions presenting as T1 benefit most from the superior precision of the digital method combined with immunohistochemical testing. The features regarding orientation, grossing, embedding and cutting the specimen remain significant, as they can considerably alter the measurements, regardless of method.

Given the importance of the Breslow thickness, the pathologist must use an accurate measurement technique for each case.

\section{Author contributions:}

Conceptualization: LA. and V.T.M..; Methodology: LA. and V.T.M..; Software: V.T.M. and L.A.; Investigation: L.A. and O.G.; Formal analysis: L.A., R.A; Statistic analysis: R.A and A.M.C. Writing - original draft preparation: LA. and V.T.M.; Writing - review and editing: D.D. and M.C.; Visualization: L.A.; Supervision: M.C. and M.S. All the authors have read and agreed with the final version of the article.

\section{Compliance with Ethics Requirements:}

„The authors declare no conflict of interest regarding this article"

"The authors declare that all the procedures and experiments of this study respect the ethical standards in the Helsinki Declaration of 1975, as revised in 2008(5), as well as the national law"

"No funding for this study”

\section{Acknowledgments:}

"The authors would like to thank the Board Committee of "Victor Babes" National Institute of Pathology, Bucharest, Romania, for their support." 


\section{References}

1. Matthews NH, Li W-Q, Qureshi AA, Weinstock MA, Cho E. Epidemiology of melanoma. In: Ward WH, Farma JM, editori. Cutaneous melanoma: etiology and therapy [Internet]. Brisbane (AU): Codon Publications; 2017. Available at: http://www.ncbi.nlm.nih.gov/books/NBK481862/. (Accessed 16 June 2019).

2. Ward WH, Lambreton F, Goel N, Yu JQ, Farma JM Clinical presentation and staging of melanoma. In: Ward WH, Farma JM, editori. Cutaneous melanoma: etiology and therapy [Internet]. Brisbane (AU): Codon Publications; 2017. Available at: http://www.ncbi.nlm.nih.gov/books/ NBK481857/ (Accessed 16 June 2019).

3. Keung EZ, Gershenwald JE. The eighth edition American Joint Committee on Cancer (AJCC) melanoma staging system: implications for melanoma treatment and care. Expert Rev Anticancer Ther. 2018;18(8):775-84.

4. Maiques O, Georgouli M, Sanz-Moreno V. Recent advances in tissue imaging for cancer research. F1000Res. 2019;8:1980.

5. Gilbertson JR, Ho J, Anthony L, Jukic DM, Yagi Y, Parwani AV. Primary histologic diagnosis using automated whole slide imaging: a validation study. BMC Clin Pathol. 2006;6(1):4.

6. Weinstein RS, Descour MR, Liang C, et al. An array microscope for ultrarapid virtual slide processing and telepathology. Design, fabrication, and validation study. Human Pathology. 2004;35(11):1303-14.

7. Jukić DM, Drogowski LM, Martina J, Parwani AV. Clinical examination and validation of primary diagnosis in anatomic pathology using whole slide digital images. Arch Pathol Lab Med. 2011;135:7.

8. Knackstedt T, Knackstedt RW, Couto R, Gastman B. Malignant melanoma: diagnostic and management update. Plastic and Reconstructive Surgery. 2018;142(2):202e-16e.
9. Lin F, Chen Z. Standardization of diagnostic immunohistochemistry: literature review and Geisinger experience. Archives of Pathology $\mathcal{E}$ Laboratory Medicine. 2014;138(12):1564-77.

10. https://www.leicabiosystems.com/digital-pathology/manage/aperio-imagescope/ (Accessed 11 October 2019).

11. Aeffner F, Zarella MD, Buchbinder N, et al. Introduction to digital image analysis in whole-slide imaging: a white paper from the Digital Pathology Association. J Pathol Inform. 2019. Available at: https://www.ncbi.nlm.nih.gov/pmc/articles/PMC6437786/ (Accessed 11 October 2019).

12. Gurcan MN, Boucheron LE, Can A, Madabhushi A, Rajpoot NM, Yener B. Histopathological image analysis: a review. IEEE Rev Biomed Eng. 2009;2:147-71.

13. Yeager K. LibGuides: SPSS tutorials: paired samples t test [Internet]. Available at: https://libguides.library.kent.edu/ SPSS/PairedSamplestTest (Accessed 11 October 2019).

14. Guo S, Zhong S, Zhang A. Privacy-preserving Kruskal-Wallis test. Comput Methods Programs Biomed. 2013;112(1):135-45.

15. Breslow A. Thickness, cross-sectional areas and depth of invasion in the prognosis of cutaneous melanoma. Ann Surg. 1970;172(5):902-8.

16. Kim JE, Chung BY, Sim CY, et al. Clinicopathologic features and prognostic factors of primary cutaneous melanoma: a multicenter study in Korea. Journal of Korean Medical Science. 2019;34(16). Available at: https://www.ncbi.nlm. nih.gov/pmc/articles/PMC6484177/ (Accessed 11 October 2019).

17. Belciu D, Patrascu OM, Neacsu F, Diaconu CC, Bodoarca S, Costache M. Skin metastases: three-year study of 50 cases in a university center. Arch Balk Med Union. 2019;54(1):97-103.

18. Stanescu R, Didilescu AC, Jianu AM, Rusu MC. Angiogenesis in the reparatory mucosa of the mandibular edentulous ridge is driven by endothelial tip cells. Rom J Morphol Embryol. 2012;53(2):375-8. 